Results Out of 284 participants data for 245 were analysed (Group 1: 27; Group 2: 107; Group 3: 111). Majority were aged 25-29 years and over 60\% had primary/lesser education. There were 39 (Group 1: 5; Group 2: 16; Group 3: 18) VFs with a total VF incidence of 8.12 [95\% CI $(5.96,11.17)]$ per 1000 Person months of observation (PMOs). Group 2 had the lowest VF incidence. Baseline CD4 $<349$ cells $/ \mathrm{mm}^{3}$ and initiation/use of TDF/3TC/EFV were associated with virologic failure (VF).

Conclusion Women at risk of VF based on the identified risk factors should be identified and targeted with appropriate intervention. Further studies are needed to verify and understand the mechanisms of association between VF and TDF/ $3 \mathrm{TC} / \mathrm{EFV}$ which is a WHO-recommended first-line ART regimen.

\section{PO 8596 ENHANCING LABORATORY DIAGNOSIS OF MYCOBACTERIUM TUBERCULOSIS IN SAMPLES FROM CHILDREN IN THE GAMBIA}

${ }^{1}$ Abigail Ayorinde* ${ }^{1}$ Edward G Coker, ${ }^{1}$ Alieu Mendy, ${ }^{1}$ Fatoumatta Cole, ${ }^{1}$ Abdou K Sillah, ${ }^{1}$ Francis S Mendy, 'Uzochukwu Egere, 1,2Beate Kampmann, 1,3 ${ }^{1}$ Leopold D Tientcheu. ${ }^{1}$ Medical Research Council Unit The Gambia at London School of Hygiene and Tropical Medicine, The Gambia; ' Imperial College, London, UK; ${ }^{3}$ Department of Biochemistry, Faculty of Science University of Yaoundé I, Yaounde, Cameroon

\subsection{6/bmjgh-2019-EDC.160}

Background Routine laboratory diagnostic methods for $M$. Tuberculosis complex (MTBC) in induced sputum samples such as smear microscopy, GeneXpert and liquid Mycobacteria growth indicator tube (MGIT) culture are often negative due to the paucibacillary nature of childhood tuberculosis. We hypothesise that prolonged incubation beyond routine culture time could potentially improve MTBC detection in specimens. Methods Out of over 1000 induced sputum samples collected during our childhood TB contact tracing research programme, we randomly selected 102 MTBC-negative MGIT cultures that had either been reported as contaminated $(n=35)$ or negative $(n=67)$ and further incubated these at $37^{\circ} \mathrm{C}$ for the duration of one month. Ziehl-Neelsen microscopy, MPT64 Antigen secretion and GeneXpert tests were repeated on all samples to detect MTBC. Bacterial DNA was extracted by CTAB method and genotyped using spoligotyping analysis.

Results Of the 1160 routinely collected induced-sputum samples $12(1 \%)$ were smear-positives; 41 (3.5\%) Xpert-positives and $51(4.4 \%)$ MGIT culture MTBC-positives. The remaining MGIT cultures were flagged as contaminated 393 (33.9\%) or MTBC-negative 644 (55.5\%). After prolonged incubation and retesting of the randomly selected ones, 26/102 (25.5\%) were now microscopy-positive, 2/55 (3.63\%) were GeneXpert-positive, 8/102 (7.8\%) MPT64-Antigen-positive, and 38/102 (37.2\%) had readable spoligotyping patterns. The predominant lineages were Mtb-Euro-American 16 (42.1\%), Mtb-Indo-Oceanic $11(28.9 \%)$ and $M$. africanum West African type-2 8 (21\%).

Conclusion Prolonged incubation of routinely MTBC-negative induced-sputum cultures yielded positive results upon retest, highlighting the low sensitivity of routine diagnosis tools on pauci-bacillary paediatric samples. Spoligotyping was more sensitive to detect MTBC compared to GeneXpert. However, prolonged incubation will cause diagnostic delays and thus better strategies are needed to improve timely childhood TB diagnosis.

\section{PO 8597 NEUTRALISING AND NON-NEUTRALISING ANTIBODIES RESPONSE IN HIV-1-INFECTED INDIVIDUALS FROM MOZAMBIQUE}

${ }^{1}$ Paloma Gonçalves*, ${ }^{2}$ Francisco Martin, ${ }^{1}$ Patricia Borges, ${ }^{3}$ Maria Espirito Santo, ${ }^{2}$ Nuno Taveira, ${ }^{1} J o s e ́$ Marcelino. ${ }^{1}$ Instituto de Higiene e Medicina Tropical (IHMT), Universidade NOVA de Lisboa, Portugal; ${ }^{2}$ Faculdade de Farmácia, Universidade de Lisboa, Lisboa, Portugali; ${ }^{3}$ Instituto do Coração, Maputo, Mozambique

\subsection{6/bmjgh-2019-EDC.161}

Background A vaccine that protects against the different HIV subtypes circulating around the world is essential to control and eliminate HIV infection. The immunogens are the key to develop an effective HIV vaccine. In this study, we characterised the antibody response against recombinant $\mathrm{C} 2 \mathrm{~V} 3 \mathrm{C} 3$ polypeptides from several HIV-1 subtypes and evaluated the neutralising antibody response.

Methods Plasmas from HIV-1-infected individuals under treatment $(n=39)$ and drugs-naïve individuals $(n=8)$ were tested in an ELISA assay to determine the presence of antibodies against polypeptides from HIV-1 subtypes (CRF02_AG, B, C, $G$ and $H$ ). The neutralising activity of plasma was evaluated with a panel of six HIV-1 viruses from a reference panel, [one tier 1 (NL4.3), and five tier 2 (PCH119_CRF07, PCE1176_C, TRO11_B, 246 F3_AC and CRF07_BJOX2000)] in a TZM-bl cells-based assay.

Results Out of 48 plasmas, $44(89.6 \%)$ reacted at least with one polypeptide and four (10.4\%) did not react with any polypeptide. Interestingly, $56 \%$ of the plasmas recognise $\geq 3$ peptides and 6 reacted with all polypeptides. The polypeptide from virus CRF02_AG was the most antigenic (77\%) followed by the polypeptide C (58.3\%), G (58.3\%), H (50\%) and B (35.4\%). There was a positive correlation between polypeptides number recognised and binding antibody reactivity $(\mathrm{r}=0.4895, \mathrm{p}=0.0111)$. All plasmas from drugs-naïve individuals neutralised at least one virus with neutralising activity between 39.3\% and 95.7\%. Four plasmas showed neutralising activity $>50 \%$ against five viruses. The virus $249 \mathrm{~F} 3$ was the easiest to neutralise (median, 65.7\%), whereas PCH119_CRF07 was the most difficult to neutralise (median, 43.6\%). Neutralising activity of plasmas from patients under treatment are ongoing.

Conclusion In summary, these polypeptides could be useful in vaccine design once they are very antigenic and we observed a heterologous neutralising antibody response in naïve patients that expressed positive antibody-response anti-peptides.

\section{PO 8607 DETECTION OF PLASMODIUM FALCIPARUM HISTIDINE- RICH PROTEIN 2/3(PFHRP-2/PFHRP-3) GENES DELETION AND AMINO ACID NUCLEOTIDE SEQUENCE VARIABILITY IN NIGERIA}

${ }^{1,2}{ }^{2}$ oland Funwei* ${ }^{1}$ Catherine 0 Falade, ${ }^{3}$ Olusola Ojurongbe. ${ }^{1}$ Department of Pharmacology and Therapeutics, University of Ibadan, Nigeria; ${ }^{2}$ Department of Pharmacy Technician Studies, Bayelsa State College of Health Technology, Nigeria; ${ }^{3}$ Department of Medical Microbiology and Parasitology, Ladoke Akintola University of Technology, Osogbo, Nigeria

\subsection{6/bmjgh-2019-EDC.162}

Background Prompt diagnosis and appropriate treatment remain the hallmark needed to reduce malaria-related mortality in areas of high transmission. Rapid diagnostic tests (RDTs) that target the Pfhrp-2 gene, are essential in resourcelimited settings where microscopy is not available. However, 